\title{
On the diversity of chemical power supply as a determinant of biological diversity
}

\author{
David Diego ${ }^{1,3}$, Bjarte Hannisdal ${ }^{1,3}$, and Håkon Dahle ${ }^{2,3,4}$ \\ ${ }^{1}$ Department of Earth Science, University of Bergen, Allégaten, NO-5007 Bergen, \\ Norway \\ ${ }^{2}$ Department of Biological Sciences, University of Bergen, Thormøhlens gate 53A, \\ NO-5006 Bergen, Norway \\ ${ }^{3}$ K.G. Jebsen Centre for Deep Sea Research, Allégaten, NO-5007 Bergen, Norway \\ ${ }^{4}$ Computational Biology Unit, Department of Informatics, University of Bergen, \\ N-5020 Bergen, Norway
}

\begin{abstract}
Understanding how environmental factors shape biological communities is a fundamental problem in microbial ecology. Patterns of microbial diversity have been characterized across a wide range of different environmental settings, but the mechanisms generating these patterns remain poorly understood. Here, we use mathematical modelling to investigate fundamental connections between chemical power supply to a system and its biological diversity and community structure. We reveal a strong mechanistic coupling between biological diversity and the diversity of chemical power supply, but also find that different properties of power supply, such as substrate fluxes and flow and Gibbs energies of reactions, affect community structure in fundamentally different ways. Moreover, we show how simple connections between power supply and growth can give rise to complex patterns of biodiversity across physicochemical gradients, such as $\mathrm{pH}$ gradients. Our findings demonstrate the importance of taking into account energy fluxes in order to reveal fundamental connections between community structure and environmental variability, and to obtain a better understanding of microbial population dynamics and diversity in natural environments.
\end{abstract}

\section{- INTRODUCTION}

2 Numerous studies have characterized microbial diversity patterns across dif-

3 ferent environmental settings. For example, $\mathrm{pH}$ has been found to be a good

4 predictor of microbial diversity in soil $[1,2]$ and temperature is correlated with 
5 marine planktonic bacterial richness on a global scale [3, 4], whereas salinity has

6 been found to be correlated with microbial diversity in lake sediments [5], soil

$7 \quad[2,6]$ and estuaries $[7,8]$.

8 However, a major challenge in the field of microbial ecology is that our un9 derstanding of the underlying dynamics generating such patterns remains very

limited [9-13]. Theoretical analyses of models representing the dynamics of highly idealized communities have provided useful insights into the conditions that favour co-existence of species, e.g. in terms of substrate uptake kinetics, [14-17], top down control by grazers [18, 19], and metabolic conversion of common substrates [11]. Clearly, however, the biodiversity, structure and functioning of microbial communities depend not only on species co-existence, but also on species abundances. Hence, the dynamics of abundance is of critical importance to any mechanistic account of how environmental conditions shape microbial communities and their activity.

At a fundamental level, all organisms have a demand for energy, or power, in order to grow and multiply. In principle, the available power supply should therefore represent a basic environmental constraint on the abundance of species. If this principle holds, then we would expect a strong coupling between power supply and diversity in most environments, especially under energy limited conditions. Indeed, recent gene-centric analyses of oxygen minimum zones have found that fluxes of energy seem to be robust predictors of microbial productivity and functional community structure [20, 21]. Moreover, in hydrothermal systems, the chemical energy landscapes emerging from mixing between reduced hydrothermal fluids and oxygenated cold seawater, seem to shape distributions of functional groups of bacteria and archaea [22, 23].

Environmental factors, such as $\mathrm{pH}$, salinity and temperature, affect the Gibbs energies of chemical reactions, and thus modulate the chemical power 
supply utilized by microbial communities [24, 25]. Part of the variation in biodiversity observed along physicochemical gradients, such as pH gradients, may therefore ultimately be linked to how those gradients affect energy landscapes.

Revealing the exact connections between chemical power supply and microbial diversity through analyses of natural environments is extremely challenging due to the high complexity and high number of unknown processes occurring in biological systems. For example, fluxes of substrate are often difficult to quantify, and extensive co-variation of variables makes it notoriously difficult to pinpoint causal effects.

An alternative to exploring natural environments is to use a theoretical modelling approach, which makes it possible to isolate the mechanistic relationship between power supply and diversity in highly idealized communities. We stress that although such models do not mimic real systems in detail, they enable us to represent basic principles in a reproducible way and to formulate testable hypotheses.

In this work, we analyse a simple population dynamics model, where growth rates are determined by maintenance powers, uptake rates of substrates, and the Gibbs energy associated with the oxidation of these substrates. We provide a thorough mathematical analysis of the relationship between biological diversity and chemical power supply in an energy limited environment. In particular, we demonstrate that complex diversity patterns along various chemical gradients can emerge from simple connections between power supply and growth. Our mathematical framework for relating chemical power supply and cellular abundances rests on fundamental thermodynamic principles. 


\section{${ }_{56}$ MATERIALS AND METHODS}

57 The model

58 We consider an idealized system, where species grow independently from each

\footnotetext{
${ }^{1}$ In order to reduce the multiplicity of constants, we take a common value for the halfsaturation constant.
}

where $r_{i}\left(\mathrm{~mol} \cdot \mathrm{s}^{-1}\right)$ denotes the maximum uptake rate and $k\left(\mathrm{~mol} \cdot \mathrm{cm}^{-3}\right)$ is the half-saturation concentration ${ }^{1}$, that is: $\rho_{i}(k)=r_{i} / 2$. Once absorbed by a cell, the $i$-th substrate $\left(S_{i}\right)$ undergoes a chemical reaction of the type $n_{i} S_{i}+a_{1} A_{1}+a_{2} A_{2}+\ldots+a_{n} A_{n} \longrightarrow b_{1} B_{1}+b_{2} B_{2}+\ldots b_{m} B_{m}$, with $A_{1}, \cdots, A_{n}$ denoting any other reactants than $S_{i}$ and $B_{1}, \cdots, B_{m}$ denoting products. The corresponding stoichiometric numbers are denoted by $n_{i}$ and $a_{1}, \cdots, a_{n}$ and $b_{1}, \cdots, b_{m}$, respectively. The Gibbs energy of the chemical reaction for each mole of the substrate of type $i, \Delta G_{r}^{i}$, in turn depends on the substrate concentration as 


$$
\Delta G_{r}^{i}=\Delta G_{i}^{0}+R T \ln Q
$$
consumer, is

where $[\cdot]$ denotes the concentration (and hence $s_{i}=\left[S_{i}\right]$ ) and $\gamma_{(\cdot)}$ is the activity coefficient for the reactant/product. Letting the activity coefficients be constant for all reactants and products, and letting the concentrations be constant for all products and reactants, except for $s_{i}$, we have that $\Delta G_{r}^{i}=$ $\Delta G_{i}^{0}-R T \ln s_{i}+K_{i}$, where $K_{i}$ is the constant $R T \ln \frac{\left(\prod \gamma_{j}\left[B_{j}\right]\right)^{b_{j} / n_{i}}}{\gamma_{i}\left(\prod \gamma_{k}\left[A_{k}\right]\right)^{a_{k} / n_{i}}}$. If we define an effective standard Gibbs energy as $\Delta G_{i \text { eff }}^{0}=\Delta G_{i}^{0}+K_{i}$, the energy available from the $i$-th reaction, which is used as an energy source by the $i$-th

$$
\begin{aligned}
& E_{i}\left(s_{i}\right)=-\Delta G_{r}^{i}=E_{i}^{0}+R T \ln s_{i}, \\
& E_{i}^{0}=-\Delta G_{i \text { eff }}^{0}>0
\end{aligned}
$$

89 The quantity $E_{i}\left(s_{i}\right)$ will be referred to as the (instantaneous) substrate$90 \quad$ specific reaction energy and hence $E_{i}^{0}$ will be called the standard substrate${ }_{91} \quad$ specific reaction energy. $E_{i}^{0}$ will be taken as

$$
E_{i}^{0}=E^{0} \mathcal{E}_{i},
$$

where $E^{0}$ can be interpreted as the basic energy scale for the considered en- 
vironment while $\mathcal{E}_{i}$ is a dimensionless factor taking into account the (possible) variability of $E_{i}^{0}$ across consumers. Although values of $E_{i}^{0}$ will rarely change with a common factor for all energy yielding reactions along a chemical gradient, settings with high $E^{0}$ can be associated with environments where negative values of $\Delta G^{0}$ are typically high. Hence, $E^{0}$ levels will typically be lower in anaerobic environments than in aerobic environments. However, the $E^{0}$ level is also influenced by the overall chemical composition of a system so that different $E^{0}$ levels may also be found along chemical gradients. As an example, consider the oxidation of lactate with sulfate as electron acceptor $\left(2\right.$ Lactate $+\mathrm{SO}_{4}^{2-} \rightarrow 2$ Acetate $+2 \mathrm{HCO}_{3}^{-}+\mathrm{H}_{2} \mathrm{~S}$ ), which has a standard Gibbs energy of $-85.3 \mathrm{~kJ} / \mathrm{mol}$ (calculated with the 'CHNOSZ' package in R [26]). Assuming that the activity coeffient of each reactant or product is one, $E^{0}$ will shift from $236 \mathrm{~kJ} / \mathrm{mol}$ in a high energy setting (acetate $=10^{-3} \mathrm{mM} ; \mathrm{HCO}_{3}^{-}=0.1 \mathrm{mM} ; \mathrm{H}_{2} \mathrm{~S}=0.1 \mathrm{mM}$; $\mathrm{SO}_{4}^{2-}=50 \mathrm{mM}$ ) to $138 \mathrm{~kJ} / \mathrm{mol}$ in a low energy setting (acetate $=50 \mathrm{mM}$; $\left.\mathrm{HCO}_{3}^{-}=20 \mathrm{mM} ; \mathrm{H}_{2} \mathrm{~S}=10 \mathrm{mM} ; \mathrm{SO}_{4}^{2-}=10 \mathrm{mM}\right)$. The same chemical variations would have similar effects on $E^{0}$ values associated with other substrates used by sulfate reducers, such as propionate, butyrate, and ethanol.

We assume in our model that all organisms have a maintenance power demand, $P_{i}$ with units $J \cdot s^{-1}$. As in several previous studies [27-29], maintenance power is defined here as the power necessary to perform all cellular processes except for growth. This includes power used in spilling reactions [30-32] and power spent on 'useful' functions (e.g. motility). How fast the population of the $i$-th species grows depends on the power available for new biomass production. This power is the difference between the substrate-consumption power $E_{i}\left(s_{i}\right) \rho_{i}\left(s_{i}\right)$, and maintenance power $P_{i}$. The rate of change in substrate concentrations in the system is defined by the flow of substrate in and out of the system, as well as the rate of consumption of the substrate. This leads to the 
following set of ODEs:

$$
\begin{aligned}
& \dot{c}_{i}=\gamma_{i}\left[E_{i}\left(s_{i}\right) \rho_{i}\left(s_{i}\right)-P_{i}\right] c_{i}, \\
& \dot{s}_{i}=\lambda\left(\phi_{i}-s_{i}\right)-\rho_{i}\left(s_{i}\right) c_{i}, \\
& 1 \leq i \leq N
\end{aligned}
$$

$121 \quad$ where $\gamma_{i}\left(J^{-1}\right)$ is the biomass yield, i.e. the amount of biomass that can be 122 built from each unit of energy. In addition, $\lambda\left(s^{-1}\right)$ is a flow rate and $\phi_{i}(m o l$. ${ }_{123} \mathrm{~cm}^{-3}$ ) is the input concentration for the $i$-th substrate. In Fig. $1 \mathrm{~b}$ we provide 124 typical values for several of the model constants. Unless otherwise specified, these values are used in the simulations. In the Supplementary Information (SI) we show the main properties of the dynamics generated by the above set of differential equations. In particular, the stationary solutions correspond to

$$
\begin{aligned}
& \gamma_{i}\left[E_{i}\left(s_{i}^{*}\right) \rho_{i}\left(s_{i}^{*}\right)-P_{i}\right] c_{i}^{*}=0, \\
& \lambda\left(\phi_{i}-s_{i}^{*}\right)-\rho_{i}\left(s_{i}^{*}\right) c_{i}^{*}=0,
\end{aligned}
$$

for all $1 \leq i \leq N$. The non trivial stationary solution $\left(c_{i}^{*}>0\right)$ requires

$$
E_{i}\left(s_{i}^{*}\right) \rho_{i}\left(s_{i}^{*}\right)=P_{i}
$$

This is a transcendental equation but one may find an explicit expression for its solution in terms of the Lambert $W$-function ${ }^{2}$ as (see SI for details)

\footnotetext{
${ }^{2}$ The Lambert $W$-function is the inverse function of $f(z)=z e^{z}$.
} 


$$
s_{i}^{*}=\frac{\frac{k P_{i}}{r_{i} R T}}{W\left(\frac{k P_{i}}{r_{i} R T} e^{\frac{r_{i} E^{0} \mathcal{E}_{i}-P_{i}}{r_{i} R T}}\right)} .
$$

The corresponding asymptotic value for the concentration of the $i$-th consumer is then

$$
c_{i}^{*}=\lambda \frac{\phi_{i}-s_{i}^{*}}{\rho_{i}\left(s_{i}^{*}\right)}=\frac{\lambda}{P_{i}} E_{e q}^{i}\left[\phi_{i}-e^{\frac{E_{e q}^{i}-E^{0} \varepsilon_{i}}{R T}}\right],
$$

where

$$
E_{e q}^{i}=E_{i}\left(s_{i}^{*}\right)=E^{0} \mathcal{E}_{i}+R T \ln s_{i}^{*},
$$

is the asymptotic value for the reaction energy corresponding to the $i$-th substrate. It can be shown that if $s_{i}^{*}<\phi_{i}$, for every $1 \leq i \leq N$, every solution to the above system with $c_{i}(0)>0$ and $s_{i}(0)>0$, for all $1 \leq i \leq N$, verifies that $\lim _{t \rightarrow \infty} c_{i}(t)=c_{i}^{*}$ and $\lim _{t \rightarrow \infty} s_{i}(t)=s_{i}^{*}$, for all $1 \leq i \leq N$ (for the formal proofs, see SI).

\section{Diversity}

Species richness is defined as the total number of species present in an ecosystem. Species evenness, on the other hand, refers to the shape of the distribution of relative abundances of the different species. The biological diversity, i.e. the $\alpha$-diversity, depends on both. Similarly, one can extend the concepts of richness, evenness and $\alpha$-diversity to taxonomic groups, genes and functional groups of organisms. Here, biological $\alpha$-diversity is defined according to the Shannon 
146 index:

$$
\begin{aligned}
& H_{B}=-\sum_{i=1}^{N} b_{i} \ln b_{i}, \\
& b_{i}=\frac{c_{i}^{*}}{\sum_{j=1}^{N} c_{j}^{*}} .
\end{aligned}
$$

The instantaneous power supply for the $i$-th consumer is defined as

$$
P_{s}^{i}(t)=\lambda \phi_{i}\left[E^{0} \mathcal{E}_{i}+R T \ln s_{i}(t)\right]
$$

148 So that

$$
\lim _{t \rightarrow \infty} P_{s}^{i}(t)=\lambda E_{e q}^{i} \phi_{i}=: P_{s}^{i}
$$

$$
\begin{aligned}
& H_{P}=-\sum_{i=1}^{N} p_{i} \ln p_{i}, \\
& p_{i}=\frac{P_{s}^{i}}{\sum_{j=1}^{N} P_{s}^{j}} .
\end{aligned}
$$

\section{${ }_{150}$ Variability of $H_{P}$ and $H_{B}$ across a chemical gradient}

The Gibbs energy of a reaction is dependent on the activities (the product of activity coefficient and concentration) of reactants and products as in equation (2). Hence, even if the concentrations of reactants and products are kept constant, the Gibbs energy of a reaction might change due to changes in activity coefficients, which are dependent on environmental factors such as salinity. If we know how concentrations and activities vary along a physicochemical gradient, we can use equation (2) to model Gibbs energies along that gradient. We 
consider here the ideal case where the concentration of one compound, acting as a substrate or product in all oxidation reactions of $s_{i}$, is fixed at different values across a series of independent systems. The activity coefficient of this compound is kept constant so that only variability in concentration causes variability in activity. As an example, we take such a compound to be $H^{+}$. We assume the fluxes of limiting substrates to be the same for all systems. Hence, we investigate how biological diversity varies along a $\mathrm{pH}$ gradient. Given a substrate concentration $s_{i}$, the reaction energy is thus given by

$$
\hat{E}_{i}\left(s_{i}\right)=E^{0} \mathcal{E}_{i}+R T \ln s_{i}+\kappa_{i} n_{i} R T p H \ln 10,
$$

where $n_{i}$ is the proton stoichiometry coefficient for the reaction of the $i$-th substrate and $\kappa_{i}$ is either +1 , if $H^{+}$is a product, or -1 if it is a reactant. The stationary solutions now depend explicitly on the $p H$, which entails the $p H$-dependence of both the biological and power supply diversities (see SI)

$$
\begin{aligned}
& H_{B}(p H)=-\sum_{i} \hat{b}_{i}(p H) \ln \left(\hat{b}_{i}(p H)\right), \\
& H_{P}(p H)=-\sum_{i} \hat{P}_{s}^{i}(p H) \ln \left(\hat{P}_{s}^{i}(p H)\right),
\end{aligned}
$$

$$
\text { with } \hat{b}_{i}(p H)=\frac{c_{i}^{*}(p H)}{\sum_{j} c_{j}^{*}(p H)} \text { and } \hat{P}_{s}^{i}(p H)=\frac{\phi_{i} \hat{E}_{e q}^{i}(p H)}{\sum_{j} \phi_{j} \hat{E}_{e q}^{j}(p H)} \text {. }
$$

\section{RESULTS}

At population equilibrium, for any given species $i$, the power supply to the system $\left(P_{s}^{i}\right)$ is determined by the flowrate $(\lambda)$, the initial substrate concentration $\left(\phi_{i}\right)$ and the reaction energy $\left(E_{e q}^{i}\right)$ (equation (14)) whereas, in terms of the power supply $\left(P_{s}^{i}\right)$, the abundance of cells for the $i$-th species $\left(c_{i}^{*}\right)$ is expressed as 


$$
c_{i}^{*}=\frac{P_{s}^{i}}{P_{i}}\left[1-\frac{1}{\phi_{i}} e^{\frac{E_{e q}^{i}-E^{0} \mathcal{E}_{i}}{R T}}\right] .
$$

Thus, the power supply does not determine uniquely the species abundance.

In order to explore the relationship between the diversity of power supply and biological diversity, we applied equations (12a) and (15a) to several combinations of $\phi_{i}, \lambda$, and $G_{e q}^{i}$ (Fig. S2).

\section{Relationships between biological diversity and parameters determining power supply}

In this section we consider the dependence of the biological diversity on the number of consumers and on the energy they are able to extract. The number of consumers will be taken to vary on the range $10-1000$. The variability in $E_{i}^{0}$, will be modelled by varying $E^{0}$ on the range $10^{3}-10^{5} \mathrm{~J} \cdot \mathrm{mol}^{-1}$. In addition, we will simulate several biologically relevant scenarios in terms of the availability of substrates and the efficiency of the consumers, as explained in the following.

Case 1: Identical power supply for all species. For reference, we first consider the trivial case where all consumers have identical traits (except for substrate specificity), and where there is no variability in input concentrations of substrates $\left(\phi_{i}\right)$ or in the molar energy available from substrate oxidation $\left(\mathcal{E}_{i}=1\right.$ for all $i$ ). Clearly, in this situation all equilibrium values will be identical, in particular given by 


$$
\begin{aligned}
& s_{i}^{*}=s^{*}\left(E^{0}\right)=\frac{\frac{k P_{0}}{r_{\max } R T}}{W\left(\frac{k P_{0}}{r_{\max } R T} e^{\frac{r_{\max } E^{0}-P_{0}}{r_{\max } R T}}\right)}, \\
& c_{i}^{*}=c^{*}=\frac{\lambda}{P_{0}} E_{e q}\left[\phi_{0}-e^{\frac{E_{e q}-E^{0}}{R T}}\right], \\
& E_{e q}=E^{0}+R T \ln s^{*}\left(E_{0}\right), \\
& \phi_{0}=1.2 \max _{E^{0}}\left\{s^{*}\left(E^{0}\right)\right\},
\end{aligned}
$$

where $\max _{E^{0}}\{\}$ denotes the maximum over the range of values considered for $E^{0}$. Therefore, the biological diversity (equation(12a)) will be just $H_{B}=\ln N$ ( $N$ being the number of consumers) and hence independent of $E^{0}$ (Fig. S3).

Case 2: Effect of variation in input concentration. In order to investigate what effect variation of input concentrations $\left(\phi_{i}\right)$ has on the biological diversity, we adjust the model from case 1 so that $\phi_{i}$ depends on the substrate-consumer pair as (Fig. S2a):

$$
\phi_{i}=\left(10^{3} e^{-\frac{(i-n / 2)^{2}}{n}}+1.2\right) \phi_{0}, \quad \phi_{0}=\max _{E^{0}}\left\{s^{*}\left(E^{0}\right)\right\}
$$

where $n$ denotes the number of consumers. Due to the symmetry of these distributions around $n / 2$, the relative abundances of consumers satisfy the constraint $b_{i}=b_{n-i}$. Since the asymptotic value for the concentration of substrates $\left(s_{i}^{*}\right)$ is independent of $\phi_{i}, s_{i}^{*}$ will in this case be the same for all $i\left(s_{i}^{*}=s^{*}\left(E^{0}\right)\right)$ while the asymptotic values of the concentrations of consumers will in general differ as 


$$
\begin{aligned}
& c_{i}^{*}\left(E^{0}\right)=\frac{\lambda}{P_{0}} E_{e q}\left[\phi_{i}-s^{*}\left(E_{0}\right)\right], \\
& E_{e q}=E^{0}+R T \ln s^{*}\left(E_{0}\right) .
\end{aligned}
$$

This is reflected in the decrease of the biological diversity magnitude with respect to the maximum value $\ln N$ ( $N$ being the number of consumers) (Fig. 2a). Notice that the relative abundance of each consumer is nearly independent of $E^{0}$ (Fig. 3a). This can be understood as follows: From equations (22), the relative abundance for the $i$-th consumer is given by

$$
\frac{c_{i}^{*}}{\sum_{j} c_{j}^{*}}=\frac{10^{3} \phi_{0} e^{-\frac{(i-n / 2)^{2}}{n}}+1.2 \phi_{0}-s^{*}\left(E^{0}\right)}{10^{3} \phi_{0} \sum_{j=1}^{n} e^{-\frac{(j-n / 2)^{2}}{n}}+n\left(1.2 \phi_{0}-s^{*}\left(E^{0}\right)\right)} .
$$

Therefore, for most of the values of $E^{0}$ the dominant term in equation (22) will be $10^{3} \phi_{0} e^{-\frac{(i-n / 2)^{2}}{n}}+1.2 \phi_{0}$ and hence the relative abundance will be nearly $E^{0}$-independent. Only for low values of $E^{0}$ is the term $s^{*}\left(E^{0}\right)$ relevant. From equation (22) it is also clear that the most abundant consumers correspond to those having the highest supply of substrates i.e. highest $\phi_{i}$ (Fig. 3a). The dependence of $E_{e q}^{i}$ on $E^{0}$ is shown in Fig. S4.

Case 3: Effect of variation in the energy scale across consumers. In order to investigate what effect variation in the energy level of substrate oxidation across consumers has on the biological diversity, we adjusted the model from case 1 so that $E^{0}$ depends on the substrate-consumer pair as (Fig. S2b)

$$
E_{i}^{0}=E^{0} \mathcal{E}_{i}, \quad \mathcal{E}_{i}=e^{-\frac{(i-n / 2)^{2}}{5 n}}+1 / 6
$$

while 


$$
\begin{aligned}
& \phi_{i}=\phi_{0}=1.2 \max _{E^{0}, i}\left\{s_{i}^{*}\left(E^{0}\right)\right\} \\
& s_{i}^{*}\left(E^{0}\right)=\frac{\frac{k P_{0}}{r_{\max } R T}}{W\left(\frac{k P_{0}}{r_{\max } R T} e^{\left.\frac{r_{\max E^{0} \mathcal{E}_{i}-P_{0}}^{r_{\max } R T}}{2}\right)}\right.} \\
& c_{i}^{*}\left(E^{0}\right)=\frac{\lambda}{P_{0}} E_{e q}^{i}\left[\phi_{0}-e^{\frac{E_{e q}^{i}-E^{0} \mathcal{E}_{i}}{R T}}\right] \\
& E_{e q}^{i}=E^{0} \mathcal{E}_{i}+R T \ln s_{i}^{*}\left(E_{0}\right)
\end{aligned}
$$

Where $\max _{E^{0}, i}\{\}$ denotes the maximum value over the range of values for $E^{0}$ and over the consumers. As expected, a gradient in the substrate reaction energy increases the sensitivity of the biological diversity on the energy scale $E^{0}$ (Fig. 2b), where a clear increase of the diversity occurs for low energy scales. The relative abundance of consumers now depends on $E^{0}$ in a non-trivial way (Fig. 3b). Notice that around $E^{0} \lesssim 3 \times 10^{4} \mathrm{~J} \cdot \mathrm{mol}^{-1}$ all consumers have almost the same abundance (and hence it corresponds to the maximum value of the biological diversity in Fig. 2b). Even though the $E^{0}$-dependence of the relative abundance is non-trivial, it still holds, as expected, that the most abundant consumers correspond to those with more availability of energy (Fig. 3b). The dependence of $E_{e q}^{i}$ on $E^{0}$ is also non-trivial in this scenario (Fig. S5).

Case 4: Effect of trade-off between energy acquisition efficiency and maintenance power. A biological trade-off between energy acquisition efficiency and maintenance power is arguably a key fitness trade-off in numerous habitats. For example, being motile by means of having flagella or having many highly efficient transporters will typically increase power demands (reducing the fitness), but at the same time increase the cellular power supply (increasing the fitness). Here, we model this trade-off by adjusting the case 1 model so that the distributions for the uptake rate $\left(r_{i}\right)$ and the maintenance power $\left(P_{i}\right)$ are given by 
(Fig. S2c and S2d)

$$
\begin{aligned}
& r_{i}=r_{\max }\left(e^{-\frac{(i-n / 2)^{2}}{n / 2}}+\frac{1}{100}\right), \\
& P_{i}=P_{0}\left(e^{-\frac{(i-n / 2)^{2}}{n / 2}}+\frac{1}{20}\right),
\end{aligned}
$$

while the remaining quantities are

$$
\begin{aligned}
& \phi_{i}=\phi_{0}=1.2 \max _{E^{0}, i}\left\{s_{i}^{*}\left(E^{0}\right)\right\}, \\
& s_{i}^{*}\left(E^{0}\right)=\frac{\frac{k P_{i}}{r_{i} R T}}{W\left(\frac{k P_{i}}{r_{i} R T} e^{\frac{r_{i} E^{0}-P_{i}}{r_{i} R T}}\right)}, \\
& c_{i}^{*}\left(E^{0}\right)=\frac{\lambda}{P_{i}} E_{e q}^{i}\left[\phi_{0}-e^{\frac{E_{e q}^{i}-E^{0}}{R T}}\right], \\
& E_{e q}^{i}=E^{0}+R T \ln s_{i}^{*}\left(E_{0}\right) .
\end{aligned}
$$

The biological diversity seems to be rather insensitive to the efficiency-cost trade-off (Fig. 2c) although the relative abundance shows a weak dependence on $E^{0}$ (Fig. 3c). It is worth noting that the most abundant consumers correspond to those with low values of both uptake-rate and maintenance power (Fig. 3c), although there is little variation between species regarding the energy available from each mole of substrate $\left(E_{e q}^{i}\right)$ (Fig. S6).

Case 5: Combined effect of biological trade-off, and variability in $\phi_{i}$ and $E_{i}^{0}$.

In order to investigate the combined affect of variations considered in cases 2-4, we modified the model from case 1 so that: 


$$
\begin{aligned}
& \phi_{i}=\left(10^{3} e^{-\frac{(i-n / 2)^{2}}{n}}+1.2\right) \phi_{0}, \quad \mathcal{E}_{i}=e^{-\frac{(i-n / 2)^{2}}{5 n}}+\frac{1}{6}, \\
& r_{i}=r_{\max }\left(e^{-\frac{(i-n / 2)^{2}}{n / 2}}+\frac{1}{100}\right), \quad P_{i}=P_{0}\left(e^{-\frac{(i-n / 2)^{2}}{n / 2}}+\frac{1}{20}\right), \\
& s_{i}^{*}\left(E^{0}\right)=\frac{\frac{k P_{i}}{r_{i} R T}}{W\left(\frac{k P_{i}}{r_{i} R T} e^{\frac{r_{i} E^{0} \mathcal{E}_{i}-P_{i}}{r_{i} R T}}\right)}, \quad \phi_{0}=\max _{E^{0}, i}\left\{s_{i}^{*}\right\}, \\
& c_{i}^{*}\left(E^{0}\right)=\frac{\lambda}{P_{i}} E_{e q}^{i}\left[\phi_{0}-e^{\frac{E_{e q}^{i}-E^{0} \mathcal{E}_{i}}{R T}}\right], \quad E_{e q}^{i}=E^{0} \mathcal{E}_{i}+R T \ln s_{i}^{*}\left(E_{0}\right) \text {. }
\end{aligned}
$$

In this case, the biological diversity acquires a non-trivial $E^{0}$-dependence with a clear increase towards low values of $E^{0}$ (Fig. 2d). The relative abundance of consumers depends on $E^{0}$ in a highly complex way (Fig. 3d). Remarkably, the particular identity of the most abundant consumer is $E^{0}$-dependent (Fig. 3d). For instance, for an energy scale of $E^{0} \sim 4 \times 10^{4} \mathrm{~J} \cdot \mathrm{mol}^{-1}$, the most abundant consumer is the one with the highest uptake rate $(i=25)$, whereas for lower energy scales $\left(E^{0} \lesssim 2 \times 10^{4} \mathrm{~J} \cdot \mathrm{mol}^{-1}\right)$ the relative abundance of the same consumer drops from $\sim 0.05$ to $\sim 0.01$, making it one of the least abundant consumers (Fig. 3d). The complex dependence of $E_{e q}^{i}$ on $E^{0}$ (Fig. S7) renders all values for $E_{e q}^{i}$ comparatively small on the range $E^{0} \sim 2-4 \times$ $10^{4} \mathrm{~J} \cdot \mathrm{mol}^{-1}$, while the energy availability is more markedly different across consumers for $E^{0} \gtrsim 4 \times 10^{4} \mathrm{~J} \cdot \mathrm{mol}^{-1}$, the species with the highest uptake rate $(i=25)$ being the most energetically advantaged. For $E^{0} \lesssim 2 \times 10^{4} \mathrm{~J} \cdot \mathrm{mol}^{-1}$ however, the $i=25$ consumer is one of the least energetically advantaged (Fig. S7). The energetic disadvantage of the $i=25$ consumer for $E^{0} \lesssim 2 \times 10^{4} J$. $\mathrm{mol}^{-1}$ is clearly reflected in its low relative abundance over these energy scales (Fig. 3d). Interestingly, for $E^{0} \gtrsim 5 \times 10^{4} \mathrm{~J} \cdot \mathrm{mol}^{-1}$, the $i=25$ consumer is not the most abundant even though it is the most energetically advantaged (i.e it has the highest $\phi_{i}$ and $\mathcal{E}_{i}$ values). This asymmetric behavior across 
energy scales clearly emerges from the combined effect of all the above model scenarios. At a high energy scale, the percentage difference between the most energetically advantaged consumers and those with baseline values for $E_{e q}^{i}$, is relatively small and of little relevance. Therefore the effect of the efficiency-cost trade-off becomes more significant. This explains why, for high energy scales, the most abundant consumers correspond to those with moderate values for both $r_{i}$ and $P_{i}$ (Fig. 3d).

\section{Relationship between biological and power supply diversi-} ties.

Here we determine how the relationship between biological diversity $\left(H_{B}\right)$ and power supply diversity $\left(H_{P}\right.$, equations $\left.(15)\right)$ is affected by distributions of $\phi_{i}$, $E_{i}^{0}$, and a biological trade-off between energy acquisition efficiency and power demands. We will consider the same distributions and combinations as above (cases 2-5).

Case 2: Effect of variability in $\phi_{i}$. We find that the relation between the biological diversity $\left(H_{B}\right)$ and the power supply diversity $\left(H_{P}\right)$ is nearly linear (because $H_{P} \simeq H_{B}$ ) across all energy scales and number of consumers considered (Fig. 4a and Fig. S8).

Case 3: Effect of variability in $E_{i}^{0}$. Considering instead distributions for $E_{i}^{0}$ as in equation (23), we find no significant deviation from the linearity relationship $H_{P} / H_{B} \simeq 1$ over all energy scales $\left(E^{0}\right)$ and number of consumers $(N)$ considered (Fig. 4b and Fig. S9).

Case 4: Effect of trade-off between energy acquisition efficiency and maintenance power. Adding a trade-off between the energy acquisition efficiency and the maintenance power increases the complexity in the relationship between $H_{B}$ and $H_{P}$, especially for low values of the number of consumers (Fig. 4c). This 
implies that the relationship between $H_{P}$ and $H_{B}$ might deviate from linearity (Fig. S10). The ratio $H_{P} / H_{B}$ is rather insensitive to the energy scale $E^{0}$.

Case 5: Combined effect of biological trade-off, and variability in $\phi_{i}$ and $E_{i}^{0}$. Similarly to the case 5 above, when all the above distributions are considered simultaneously, the complexity of the relation between the biological diversity and the power supply diversity increases significantly (Fig. 4d and Fig. S11). It is worth noting that each of the above considered scenarios by itself renders the power diversity always greater than the biological diversity $\left(H_{P} / H_{B} \geq 1\right)$. However, when all these scenarios are considered simultaneously, the biological diversity can become significantly greater than the power diversity (Fig. 4).

\section{Global scaling of the power supply}

Increasing the overall power supply $\left(\sum_{i} \lambda \phi_{i} E_{e q}^{i}\right)$ by increasing the flow of fluids into the system (i.e. increasing $\lambda$ ), has no effect on $H_{B}$. This is evident, as $\lambda$ factors out in the calculation of the relative abundance of a species (equation (12b)). However, changing the concentration of all substrates in the fluids entering the system has an effect on $H_{B}$, even when the diversity of power supply $H_{P}$ remains unaffected. To see this, we analysed the response of the biological diversity to a global scaling of the power supply, i.e. $P_{s}^{i} \rightarrow \Lambda P_{s}^{i}$, Fig. 5. In particular, under a global rescaling of the initial concentration of substrate as $\phi_{i}(\Lambda)=\Lambda \phi_{i}$ (the Gibbs energy at equilibrium population is unaffected by such a scaling), the relative abundance of specialists (equation (12b)) is modified to

$$
b_{i}(\Lambda)=\frac{A_{i}-B_{i} / \Lambda}{A-B / \Lambda}
$$

where $A_{i}=\frac{E_{e q}^{i} \phi_{i}}{P_{i}}, B_{i}=\frac{E_{e q}^{i}}{P_{i}} s_{i}^{*}, A=\sum_{i} A_{i}$ and $B=\sum_{i} B_{i}$. Therefore, 
for high enough values of the scaling factor it holds that $H_{B}(\Lambda)$ saturates to $\ln (A)-\frac{1}{A} \sum_{i} A_{i} \ln \left(A_{i}\right)$ (Fig. 5). This demonstrates that changing the total power supply to the system, may or may not affect $H_{B}$, depending on the exact setting of the system and whether the change in power supply is a result of changes in fluid flow or a scaling of the concentration of substrates entering the system.

\section{Biological diversity dependence on $\mathrm{pH}$}

How the biological diversity changes with $\mathrm{pH}$ is, within our modelling framework, largely dependent on the exact chemical and biological setting of the system. For example, in a system with only two biological species where $H^{+}$ is produced by one organism and consumed by the other, a decrease in $\mathrm{pH}$ (i.e. increase in $\mathrm{H}^{+}$) causes an increase of the power supply to the consumer of $\mathrm{H}^{+}$whereas it makes the power supply to the producer decrease. This is easily checked using equation (16), from which we readily see that $\frac{\partial E_{i}}{\partial p H}<0$ for the consumer while $\frac{\partial E_{i}}{\partial p H}>0$ for the producer. Depending on the particular values of $\Delta G^{0}$ for the chemical reactions used by the two biological species, the available energies at population equilibrium $\left(E_{e q}^{i}\right)$ may diverge from each other (Fig. S12a) or converge to each other and cross (Fig. 6c). This reflects on the corresponding stationary concentrations of both species (Fig. 6a). The effect on the biological diversity is either a monotonic decrease (Fig. S13b) or the presence of a global maximum (Fig. 6b). In a system with many biological species, such connections may become highly complex (Fig. 6e - 6h). In particular, even when the shape of the power supply diversity is essentially the same as that for very few species (Fig. 6h and 6d), the corresponding biological diversity displays a highly complex dependence on the $\mathrm{pH}$ (Fig. 6f). These analyses demonstrate how $H_{B}$ can vary along a $\mathrm{pH}$ gradient, due to a ther- 


\section{${ }_{352}$ DISCUSSION}

modynamic dependency between $\mathrm{pH}$ and power supply. Within our modelling framework, the connections between $\mathrm{pH}$ and diversity will be similar between systems hosting the same biological species, but can be very different between systems hosting different biological species.

This study provides a comprehensive theoretical analysis of the coupling between fluxes of chemical energy and $\alpha$-diversity. We consider a population dynamic model where growth is energy limited, which arguably is the case for most of Earth's biosphere [33]. Our model is derived from a few fundamental principles relating chemical power supply to a system, cellular rates of substrate uptake, cellular power demands, and population size. The model assumes that biological species grow independently of each other on one limiting substrate each, hence the species richness is trivially equal to the number of limiting substrates. However, by shaping the relative abundance of species, fluxes of energy influence the biodiversity in non-intuitive ways.

The model parameters have a clear relevance to real ecosystems. For example, $\lambda$ may describe the flow rate of substrates into and out of a fermentor or river discharge into and out of a lake; values of $\phi_{i}$ describe concentrations of substrate in the inflow; $E^{0}$ levels describe typical energy availability per mole of substrate oxidation under given environmental conditions, and $E_{i}^{0}$ values describe cell-specific energy availability per mole of substrate. This study demonstrates that even within a simple and highly idealised model framework, complex relationships emerge between the energetic setting of a system and its biodiversity where distributions of $\phi_{i}$ and $E_{i}^{0}$, as well as $E^{0}$ levels, contribute to shaping biodiversity in distinct ways. Adding a biological trade-off between energy acquisition efficiency and maintenance power increases this complexity 
even further.

Our numerical experiments demonstrate that a global scaling of $E^{0}$ is sufficient to create changes in diversity patterns. Interestingly, $E^{0}$ levels also seem to have a large impact on the identity of dominant species in models where both $E_{i}^{0}$ and $\phi_{i}$ values vary and a trade-off between $P_{i}$ and $r_{i}$ is considered (Fig. $3 \mathrm{~d}$ ). Although values of $E^{0}$ will rarely change with a common factor for all energy yielding reactions along a chemical gradient, the energy scale $E^{0}$ is a potentially important parameter for understanding how environmental conditions shape the overall distribution of microbial species. Changing the power supply to a system by a scaling of $\lambda$ has a fundamentally different effect on $H_{B}$ than if the same increase in power supply occurs due to a global scaling of $\phi_{i}$ values - i.e. within our modelling framework, $H_{B}$ remains unaffected by a scaling of $\lambda$ but responds to a scaling of $\phi_{i}$ values, particularly for low $E^{0}$ values Fig. 5. This finding has a clear relevance to natural systems. For example, if we want to predict the microbial diversity in an ecosystem, then the concentration of substrates in fluids flowing into the system may be a stronger predictor than the rate of fluid inflow. Note that variability in $\phi_{i}$ does not affect the chemical composition of the system (except for species abundance). Consequently, environments with identical in situ environmental conditions may still host microbial communities with different $H_{B}$ due to differences in the mode of power supply.

Despite the emergent complexity of the connections between energy supply and diversity, our results suggest that the diversity of power supply $\left(H_{P}\right)$ may be an overall good predictor for biological diversity $\left(H_{B}\right)$, at least across environments with similarly shaped distributions of $\phi_{i}$ and $E_{i}^{0}$ values (Fig. S8-S11). Whether or not such connections hold when more complex food webs and species interactions are considered, is clearly a topic for future research. We stress, however, that a strong correlation between $H_{P}$ and $H_{B}$ does not imply that chemical 
gradients shape biodiversity patterns in a simple way. Rather, as exemplified by our analysis of biodiversity along a pH gradient (Fig. 6), variations in the activity of a single chemical compound may have very different effects on $H_{P}$ and $H_{B}$ under different chemical and biological settings. Such heterogeneity in the relationship between $\mathrm{pH}$ and microbial $\alpha$-diversity has been observed in different environments. In a study of 431 geographically widespread and environmentally disparate lakes, no correlation was found between $\alpha$-diversity and $\mathrm{pH}$ [34]. In contrast, $\mathrm{pH}$ has been found to be a major driver of soil communities and is often reported to be one of the strongest predictors of $\alpha$-diversity [1, 35]. Reported trends in the relationship between $\mathrm{pH}$ and microbial $\alpha$-diversity in soil also differ. In an analysis of 300 grassland and forest soils in Germany, $\alpha$-diversity increased with $\mathrm{pH}$ from $\mathrm{pH} 3$ to $\mathrm{pH} 7.5$, but with a plateau around $\mathrm{pH} 5-6$ [1]. In analyses of numerous types of US soil samples, covering a $\mathrm{pH}$ range of 3-9, the $\alpha$-diversity peaked at $\mathrm{pH}$ around $6-7$. The diversity patterns observed in soils globally seem to emerge from an aggregation of multiple simpler relationships between $\mathrm{pH}$ and the relative abundance of individual taxonomic groups from phylum to species level [1,36-38]. Intriguingly, this emergence of complexity from simple $\mathrm{pH}$ dependence of species abundance is what we find in our model (Fig. 6e,f).

Based on our modelling results, we propose three expectations that can act as working hypotheses for further inquiry:

- $E^{0}$ levels and the shape of the distributions of $\phi_{i}$ and $E_{i}^{0}$ influence microbial biodiversity in different ways. $H_{B}$ is more sensitive to variation in the $E_{i}^{0}$ distribution than to comparable variation in the distribution of $\phi_{i}$ values.

- $H_{P}$ is a useful predictor for $H_{B}$ across environments with similar $E^{0}$ levels and similarly shaped distributions of $\phi_{i}$ and $E_{i}^{0}$. 
- There is no general trend between a given chemical gradient and biodiversity, rather the relationship between them depends on the thermodynamic setting of the environment.

These expectations can be tested directly under chemostat conditions where chemical fluxes and the chemical composition of the system can be controlled, and microbial communities can be easily monitored - e.g. through 16S rRNA gene sequence analyses. In order to set up an experimental system comparable to what is modelled here, the species grown in the chemostat should have distinct substrate spectra so that each species acquires energy by the oxidation of one limiting substrate each. In principle, one could analyse diversity patterns in a system with only two species, but a higher number of species may be desirable for a more robust analysis. Estimates of maintenance power can be obtained experimentally, taking into account that maintenance power depends on environmental conditions, such as temperature [33, 39, 40].

In the field of microbial ecology, connections between environmental setting and biodiversity in natural systems have thus far mostly been explored through linear regression analyses or multivariate analyses involving directly measurable environmental parameters. Our results suggest that in order to identify driving mechanisms of biodiversity and community structure, a concerted effort should be put into assessing the role of power supply. Quantifying chemical power supply in natural environments can be challenging as it requires accurate information on chemical composition and dominant chemical fluxes in the system. Another complicating factor is that variations in the concentration of a chemical compound may have both direct and indirect effects on energy fluxes. For example, $\mathrm{pH}$ influences energy availability directly in energy yielding reactions where protons act as reactants or products, but also indirectly by modulating the activity coefficient or chemical speciation of numerous chemical compounds [24]. 
Hence, there is a need to develop improved methods for estimating energy fluxes and including such estimates in ecological studies to test model predictions.

In summary, our findings highlight the importance of taking into account energy supply and energy utilization in microbial systems in order to advance our understanding of how the fundamental laws of thermodynamics shape the biosphere.

\section{References}

1. Kaiser, K. et al. Driving forces of soil bacterial community structure, diversity, and function in temperate grasslands and forests. Scientific Reports 6. ISSN: 2045-2322 (SEP 21 2016).

2. Zhao, S. et al. Soil $\mathrm{pH}$ is equally important as salinity in shaping bacterial communities in saline soils under halophytic vegetation. Scientific Reports 8. ISSN: 2045-2322 (MAR 14 2018).

3. Pommier, T. et al. Global patterns of diversity and community structure in marine bacterioplankton. Molecular Ecology 16, 867-880. ISSN: 0962-1083 (FEB 2007).

4. Fuhrman, J. A. Microbial community structure and its functional implications. Nature 459, 193-199. ISSN: 0028-0836 (MAY 14 2009).

5. Yang, J., Ma, L., Jiang, H., Wu, G. \& Dong, H. Salinity shapes microbial diversity and community structure in surface sediments of the QinghaiTibetan Lakes. Scientific Reports 6. ISSN: 2045-2322 (APR 26 2016).

6. Zhang, K. et al. Salinity Is a Key Determinant for Soil Microbial Communities in a Desert Ecosystem. mSystems 4. ISSN: 2379-5077 (JAN-FEB 2019). 
7. Vidal-Dura, A., Burke, I. T., Mortimer, R. J. G. \& Stewart, D. I. Diversity patterns of benthic bacterial communities along the salinity continuum of the Humber estuary (UK). Aquatic Microbial Ecology 81, 277-291. ISSN: 0948-3055 (2018).

8. Osterholz, H., Kirchman, D. L., Niggemann, J. \& Dittmar, T. Diversity of bacterial communities and dissolved organic matter in a temperate estuary. FEMS Microbiology Ecology 94. ISSN: 0168-6496 (AUG 2018).

9. Antwis, R. E. et al. Fifty important research questions in microbial ecology. FEMS Microbiology Ecology 93. ISSN: 0168-6496 (MAY 2017).

10. Grosskopf, T. \& Soyer, O. S. Synthetic microbial communities. Current Opinion in Microbiology 18, 72-77. ISSN: 1369-5274 (2014).

11. Grosskopf, T. \& Soyer, O. S. Microbial diversity arising from thermodynamic constraints. English. ISME Journal 10, 2725-2733. ISSN: 1751-7362 (NOV 2016).

12. Gudelj, I. et al. An integrative approach to understanding microbial diversity: from intracellular mechanisms to community structure. Ecology Letters 13, 1073-1084. ISSN: 1461-023X (SEP 2010).

13. O'Brien, S., Hodgson, D. J. \& Buckling, A. The interplay between microevolution and community structure in microbial populations. Current Opinion in Biotechnology 24, 821-825. ISSN: 0958-1669 (AUG 2013).

14. Hsu, S.-B., Hubbell, S. \& Waltman, P. A mathematical theory for singlenutrient competition in continuous cultures of micro-organisms. SIAM Journal on Applied Mathematics 32, 366-383 (1977).

15. Hsu, S. Limiting behavior for competing species. SIAM Journal on Applied Mathematics 34, 760-763 (1978). 
16. Butler, G. \& Wolkowicz, G. A mathematical model of the chemostat with a general class of functions describing nutrient uptake. SIAM Journal on Applied Mathematics 45, 138-151 (1985).

17. Wolkowicz, G. S. \& Lu, Z. Global dynamics of a mathematical model of competition in the chemostat: general response functions and differential death rates. SIAM Journal on Applied Mathematics 52, 222-233 (1992).

18. Winter, C., Bouvier, T., Weinbauer, M. G. \& Thingstad, T. F. Trade-Offs between Competition and Defense Specialists among Unicellular Planktonic Organisms: the "Killing the Winner" Hypothesis Revisited. Microbiology and Molecular Biology Reviews 74, 42-57. ISSN: 1092-2172 (MAR 2010).

19. Thingstad, T. Elements of a theory for the mechanisms controlling abundance, diversity, and biogeochemical role of lytic bacterial viruses in aquatic systems. Limnology and Oceanography 45, 1320-1328. ISSN: 0024-3590 (SEP 2000).

20. Louca, S. et al. Integrating biogeochemistry with multiomic sequence information in a model oxygen minimum zone. Proceedings of the National Academy of Sciences of the United States of America 113, E5925-E5933. ISSN: 0027-8424 (OCT 4 2016).

21. Reed, D. C., Algar, C. K., Huber, J. A. \& Dick, G. J. Gene-centric approach to integrating environmental genomics and biogeochemical models. Proceedings of the National Academy of Sciences of the United States of America 111, 1879-1884. ISSN: 0027-8424 (FEB 4 2014).

22. Dahle, H., Okland, I., Thorseth, I. H., Pederesen, R. B. \& Steen, I. H. Energy landscapes shape microbial communities in hydrothermal systems on the Arctic Mid-Ocean Ridge. ISME Journal 9, 1593-1606. ISSN: 17517362 (JUL 2015). 
23. Dahle, H. et al. Energy Landscapes in Hydrothermal Chimneys Shape Distributions of Primary Producers. Frontiers in Microbiology 9. ISSN: 1664302X (JUL 16 2018).

24. Jin, Q. \& Kirk, M. F. pH as a Primary Control in Environmental Microbiology: 1. Thermodynamic Perspective. Frontiers in Environmental Science 6. ISSN: 2296-665X (MAY 1 2018).

25. Larowe, D. E. \& Amend, J. P. Catabolic rates, population sizes and doubling/replacement times of microorganisms in natural settings. American Journal of Science 315, 167-203. ISSN: 0002-9599 (MAR 2015).

26. Dick, J. M. CHNOSZ: Thermodynamic calculations and diagrams for geochemistry. Frontiers in Earth Science 7, 180 (2019).

27. Pirt, S. J. The maintenance energy of bacteria in growing cultures. Proc. R. Soc. Lond., B, Biol. Sci. 163, 224-231 (Oct. 1965).

28. Hoehler, T. M. \& Jorgensen, B. B. Microbial life under extreme energy limitation. Nat. Rev. Microbiol. 11, 83-94 (Feb. 2013).

29. Van Bodegom, P. Microbial maintenance: a critical review on its quantification. Microb. Ecol. 53, 513-523 (May 2007).

30. Neijssel, O. M. \& Tempest, D. W. The role of energy-spilling reactions in the growth of Klebsiella aerogenes NCTC 418 in aerobic chemostat culture. Arch. Microbiol. 110, 305-311 (Nov. 1976).

31. Cook, G. M. \& Russell, J. B. Energy-spilling reactions of Streptococcus bovis and resistance of its membrane to proton conductance. Appl. Environ. Microbiol. 60, 1942-1948 (June 1994).

32. Russell, J. B. \& Strobel, H. J. ATPase-dependent energy spilling by the ruminal bacterium, Streptococcus bovis. Arch. Microbiol. 153, 378-383 (1990). 
33. Hoehler, T. M. Biological energy requirements as quantitative boundary conditions for life in the subsurface. Geobiology 2, 205-215. ISSN: 1472-4677 (OCT 2004).

34. Yang, J., Jiang, H., Dong, H. \& Liu, Y. A comprehensive census of lake microbial diversity on a global scale. Science China-Life Sciences 62, 13201331. ISSN: 1674-7305 (OCT 2019).

35. Fierer, N. \& Jackson, R. The diversity and biogeography of soil bacterial communities. Proceedings of the National Academy of Sciences of the United States of America 103, 626-631. ISSN: 0027-8424 (JAN 17 2006).

36. Nacke, H. et al. Pyrosequencing-Based Assessment of Bacterial Community Structure Along Different Management Types in German Forest and Grassland Soils. PLOS ONE 6. ISSN: 1932-6203 (FEB 16 2011).

37. Will, C. et al. Horizon-Specific Bacterial Community Composition of German Grassland Soils, as Revealed by Pyrosequencing-Based Analysis of 16S rRNA Genes. Applied and Environmental Microbiology 76, 6751-6759. ISSN: 0099-2240 (OCT 2010).

38. Lauber, C. L., Hamady, M., Knight, R. \& Fierer, N. Pyrosequencing-Based Assessment of Soil pH as a Predictor of Soil Bacterial Community Structure at the Continental Scale. Applied and Environmental Microbiology 75, 5111-5120. ISSN: 0099-2240 (AUG 1 2009).

39. Tijhuis, L., Van Loosdrecht, M. C. M. \& Heijnen, J. J. A thermodynamically based correlation for maintenance gibbs energy requirements in aerobic and anaerobic chemotrophic growth. Biotechnology and Bioengineering 42, 509-519. eprint: https://onlinelibrary.wiley.com/doi/pdf/10. 1002/bit. 260420415. https : //onlinelibrary.wiley.com/doi/abs / 10.1002/bit.260420415 (1993). 


\section{${ }_{590}$ Competing interests} 0027-8424 (MAR 30 2004).

\section{Acknowledgements}

40. Price, P. \& Sowers, T. Temperature dependence of metabolic rates for microbial growth, maintenance, and survival. Proceedings of the National Academy of Sciences of the United States of America 101, 4631-4636. ISSN:

This work was supported by the K.G. Jebsen Center for Deep Sea Research and by a Trond Mohn Foundation Starting Grant to B.H.

The authors declare no competing interests. 
(a)

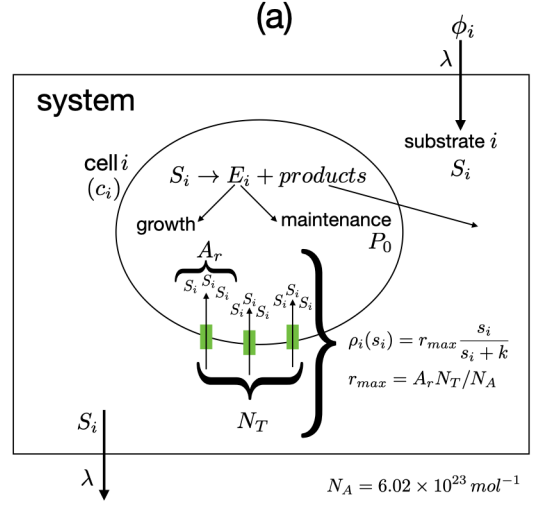

(b)

\begin{tabular}{|cccc|}
\hline Symbol & Parameter & Magnitude & Units \\
$P_{0}$ & maintenance power & $10^{-13}$ & $\frac{J}{s \cdot c e l l}$ \\
$N_{T}$ & $\sharp$ transporters & $10^{4}$ & - \\
$A_{r}$ & max. absorp. rate & $10^{4}$ & $\frac{\text { molecule }}{s \cdot \text { transporter }}$ \\
$k$ & saturation const. & $10^{-6}$ & $\frac{\text { mol }}{\mathrm{cm}^{3}}$ \\
$\lambda$ & flowrate & $10^{-8}$ & $\mathrm{~s}^{-1}$ \\
$r_{\max }$ & max. uptake rate & $10^{-16}$ & $\frac{\mathrm{mol}}{\mathrm{s}}$ \\
\hline
\end{tabular}

Figure 1: (a) Model Schematic: Substrates flow into and out of a system with a fixed flowrate $(\lambda)$. The power supply of the $i$-th substrate is defined as the product substrate specific inflow concentration $\left(\phi_{i}\right)$, the flowrate $(\lambda)$ and the energy available from each mole of substrate $\left(E_{i}\right)$. Once the $i$-th substrate enters the system it is homogenously distributed in the system to the concentration $s_{i}$. The $i$-th biological species consumes the $i$-th substrate only, and at a rate $\left(\rho_{i}\right)$ dependent on $s_{i}$, modelled according to Michaelis-Menten kinetics in equation (1), so that the uptake of the $i$-th substrate by the $i$-th species is the product between $\rho_{i}$ and the total abundance of the $i$-th species $\left(c_{i}\right)$. The cell specific power supply is the product $E_{i} \rho_{i}$. Cellular growth rates depend on the power available for growth after a fixed amount of power has been used for maintenance (equation (6a)). The maximum uptake rate is a derived constant obtained as $r_{\max }=N_{T} A_{r} / N_{A}$, with $N_{A}$ denoting the Avogadro number.

(b) Model parameter values: Model constant values used in this work. 
a

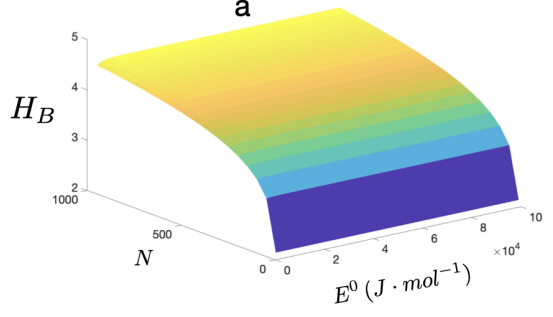

C

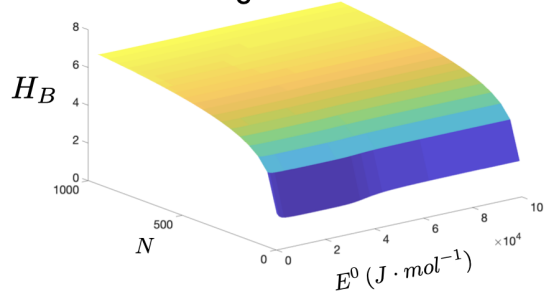

b

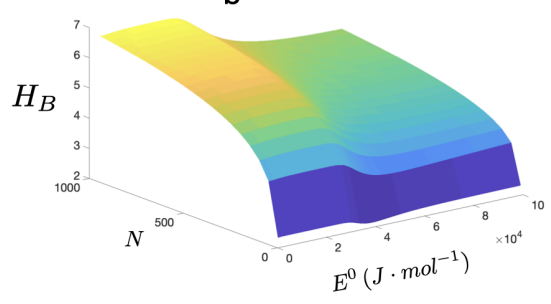

d

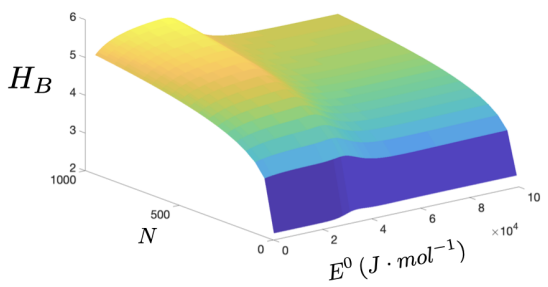

Figure 2: Biological diversity $\left(H_{B}\right)$ as a function of the number of consumers $(N)$ and $E^{0}$. The graphic a shows $H_{B}$ corresponding to a distribution of $\phi_{i}$ given by equation (20) and model parameters as in equations (21) ; Graphic b shows $H_{B}$ corresponding to a distribution of $E_{i}^{0}$ as in equation (23) and model parameters as in equations (24) ; Graphic c shows $H_{B}$ corresponding to distributions of $r_{i}$ and $P_{i}$ as in equations (25) and model parameters as in equations (26) ; The graphic $\mathbf{d}$ shows the biological diversity when all the previous distributions are considered simultaneously (model parameters as in equations (27)).
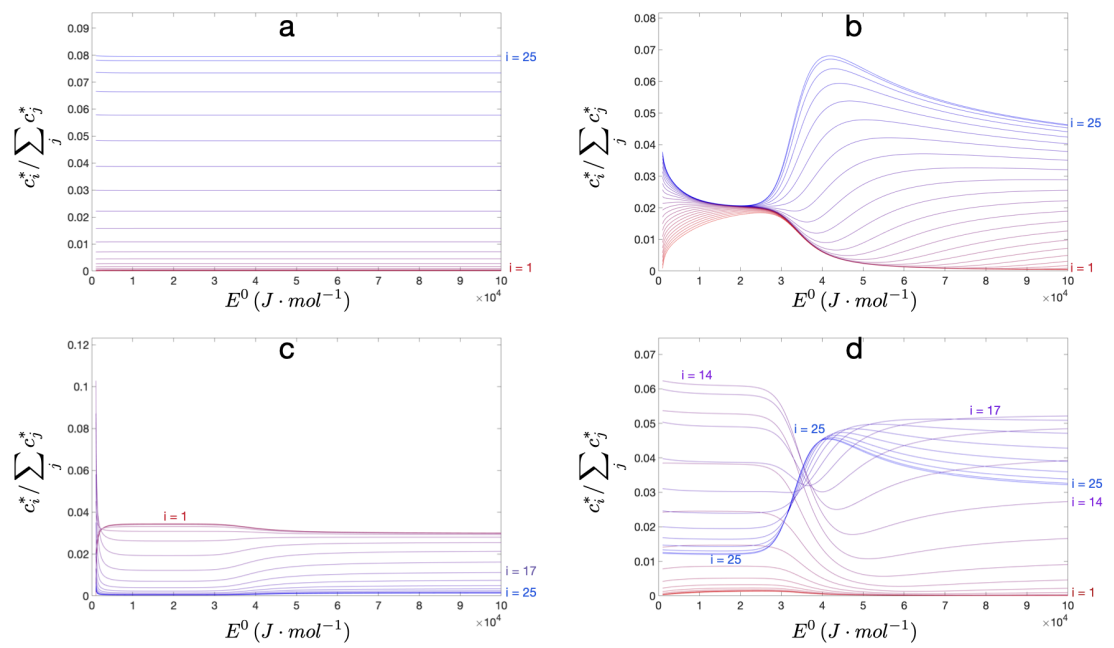

Figure 3: Relative abundance of cells as a function of $E^{0}$ for $N=50$ consumers. The graphics show the relative abundance corresponding to the model parameters used to produce Fig. 2a, 2b, 2c and 2d, respectively. Due to symmetry, only species labeled 1-25 are shown. The color gradient indicates species label (red - species 1; blue - species 25). 

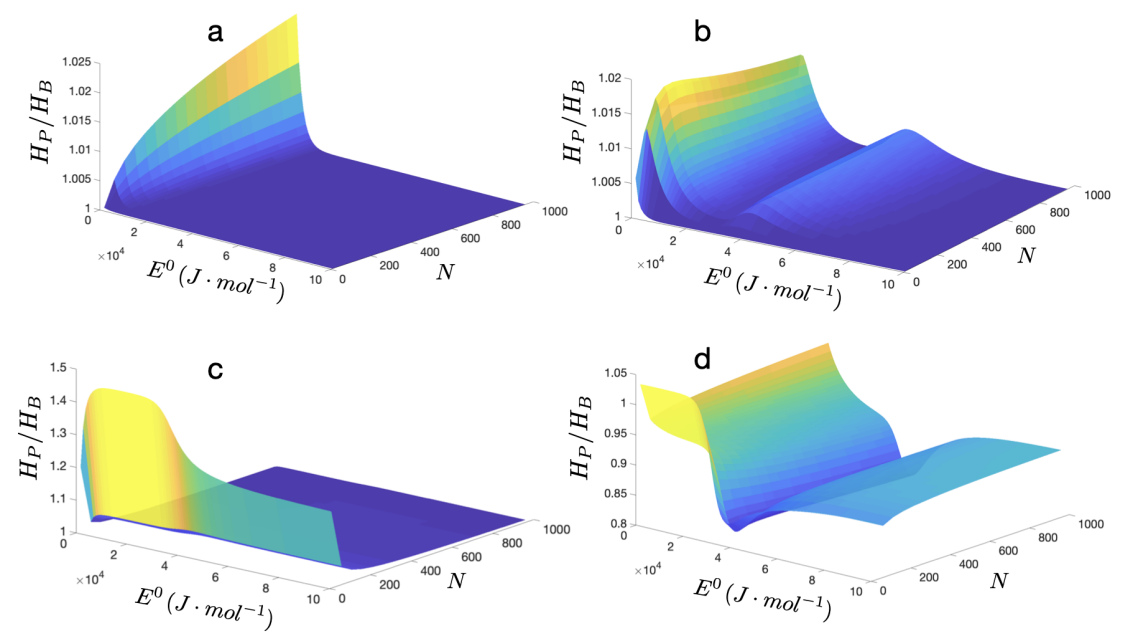

Figure 4: Ratio between power diversity $\left(H_{P}\right)$ and biological diversity $\left(H_{B}\right)$ as a function of the number of consumers $(N)$ and the energy scale $\left(E^{0}\right)$. The graphics show the ratio $H_{P} / H_{B}$ obtained with the model parameters used to produce Fig. $2 \mathrm{a}, 2 \mathrm{~b}, 2 \mathrm{c}$ and $2 \mathrm{~d}$, respectively.

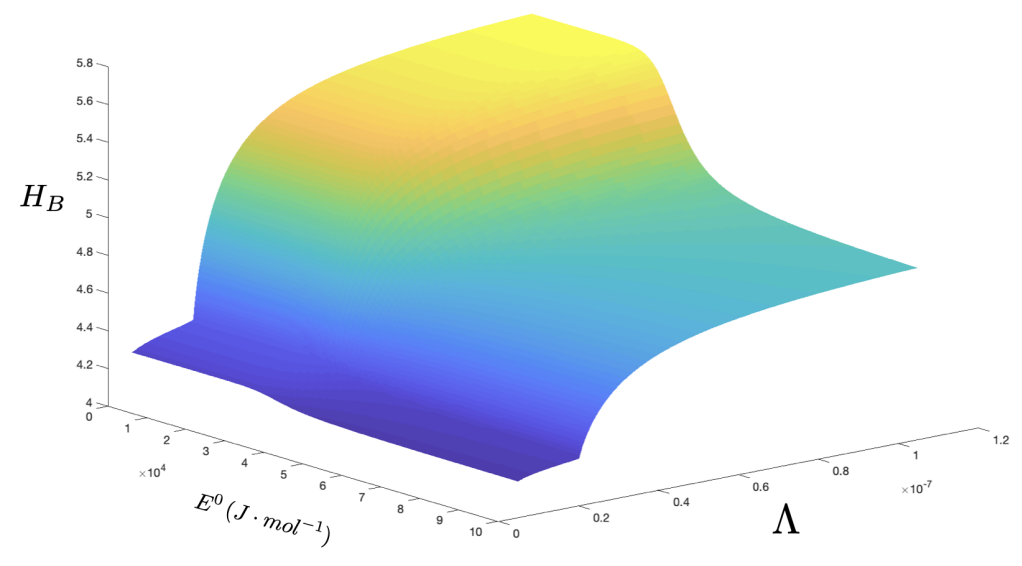

Figure 5: Biological diversity as a function of the energy scale $\left(E^{0}\right)$ and a global scaling of the input substrate concentration (i.e. $\phi_{i} \mapsto \Lambda \phi_{i}$ ). The number of specialists is set to $n=500$. We consider distributions for $\phi_{i}, r_{i}$ and $P_{i}$ given by $\phi_{i} / \phi_{0}=10^{3} e^{-\frac{(i-n / 2)^{2}}{n / 2}}+1.2$, $r_{i} / r_{\max }=e^{-\frac{(i-n / 2)^{2}}{n / 2}}+1 / 100$ and $P_{i} / P_{0}=e^{-\frac{(i-n / 2)^{2}}{n / 2}}+1 / 20$. The stoichiometric coefficient is set to 5 for each substrate and the temperature is set to $T=300 \mathrm{~K}$. The remaining parameters are given the values in Fig. 1b. 

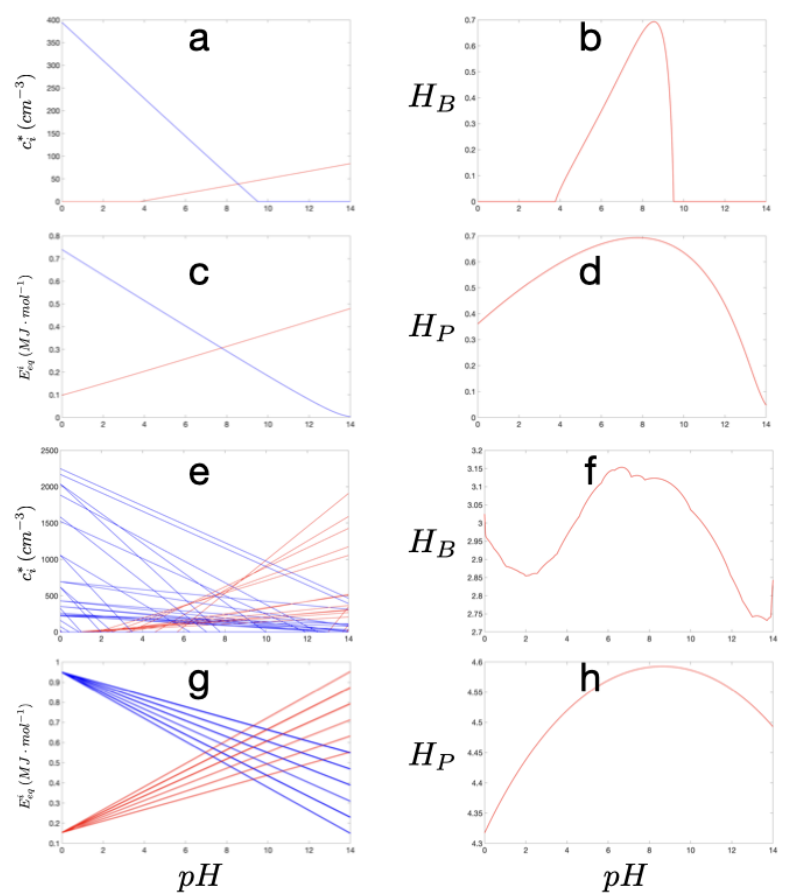

Figure 6: Effect of $p H$ on biological and power supply diversities. The energy scale is set to $E^{0}=10^{6} \mathrm{~J} \cdot \mathrm{mol}^{-1}$ and the temperature to $T=300 \mathrm{~K}$. We consider a trade-off between uptake and power maintenance as given in equations (25) while all substrates have the same input concentration given by $\phi_{0}=5 \frac{\frac{k P_{0}}{r_{\max R T}}}{W\left(\frac{k P_{0}}{r_{\max R T}} e^{\frac{r_{\max } E^{0}-P_{0}}{r_{\max R T}}}\right)}$, where $W(z)$ is the Lambert $W$-function. The remaining parameters are set to the values in Fig. $1 \mathrm{~b}$.

The graphic a shows the abundance of cells for the case of two specialists where one of them is an $\mathrm{H}^{+}$-producer (red line) and the other is an $\mathrm{H}^{+}$-consumer (blue line). The stoichiometric coefficients are set as 5 for the producer and 10 for the consumer; The graphic $\mathbf{b}$ shows the corresponding biological diversity ; Graphic c shows the $p H$-dependence of $E_{e q}^{i}$ corresponding to the plot $\mathbf{a}$. The red line shows $E_{e q}$ for the $H^{+}$-producer and the blue line corresponds to the $H^{+}$-consumer ; The plot $\mathbf{d}$ shows the corresponding power supply diversity $\left(H_{P}\right)$; The graphics $\mathbf{e}, \mathbf{f}, \mathbf{g}$ and $\mathbf{h}$ show the same as $\mathbf{a}, \mathbf{b}, \mathbf{c}$ and $\mathbf{d}$, respectively, but for 100 specialists. Half of them (chosen randomly) are set as $\mathrm{H}^{+}$-consumers (red lines) and the other half as $H^{+}$-producers (blue lines). The stoichiometric coefficients vary between 5 and 10 and each specialist is randomly assigned a number within this interval. 